\title{
Critique de livre
}

Mahler, Jean (2018):

Anne- une mort choisie

Lausanne: Edition Ouverture (Collection «son mot à dire ...»),104 pages, 15,00 francs suisses

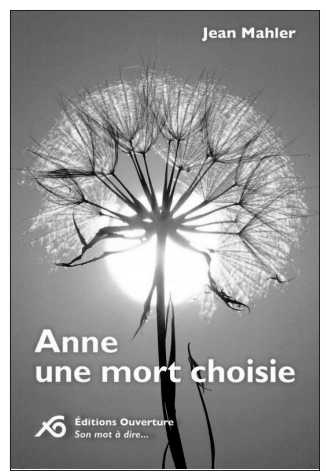

Anne est la fille de Jean Mahler. À l'âge de 43 ans, elle a perdu son compagnon à la suite d'une longue maladie et apprend un an plus tard qu'elle est elle-même atteinte de cancer et qu'il n'y a aucun espoir de guérison. Tous deux étaient médecins.

D’une manière impressionnante, Jean Mahler décrit du point de vue du père, respectivement des parents, comment s'est développée la confrontation avec la maladie. Celle-ci aboutit au final à la décision de la fille de faire appel à Exit et de choisir elle-même le moment de la mort afin de ne pas devoir s'y opposer dans des douleurs de plus en plus importantes. Elle accepte sa mort inéluctable, prend en main ce qu'elle peut encore choisir elle-même et conserve ainsi sa dignité. Ce qui est impressionnant c'est la façon dont les médecins consultés ont proposé différentes thérapies en sachant qu'aucune ne serait fructueuse et quau final ils confessent pouvoir comprendre la décision d'Anne. Mais, aucun d'entre eux n'est prêt à l'accompagner sur sa voie. Seul son psychothérapeute y consent - et ses parents - avec qui elle vit depuis la mort de son conjoint. Jean Mahler décrit en détail, dans un langage clair et délicat, le processus qu'ils ont tous les trois traversé, comment ils ont traversé ensemble les phases du deuil, comme ils sont parvenus, et sa sœur aussi, à se dire adieu, à mettre fin à la vie jusquà l'accompagnement au moment de l'arrivée de la mort.

Lauteur écrit sur la mort qui est une partie de la vie. Souvent, ce sujet est aujourd'hui encore tabou (en particulier la mort choisie). Il faut remercier l'auteur et la maison d'édition qui ont permis qu'un sujet émouvant puisse être rendu public et discuté avec cette publication. Lécriture a certainement aussi servi à ce que le père gère la mort de la fille, mais la publication de ce destin individuel ouvre aussi une dimension universelle et réveille des résonances, la sensation dêtre touché et la réflexion auprès des lectrices et lecteurs. Jean Mahler est psychothérapeute et membre de l'ASP. Dans l'un des derniers chapitres, il décrit comment l'annonce de la mort de sa fille et l'accompagnement par le père ont eu un effet sur chacune de ses thérapies et ont donné lieu à des séances très touchantes et approfondies.

Merci Jean de partager avec nous cette expérience. Je recommande la lecture de ce livret (format : $11 \times 16,2 \mathrm{~cm}$ ). Il est écrit en français, mais les collègues de langue allemande et italienne peuvent également le lire, idéalement avec quelques connaissances scolaires en français.

Peter Schulthess 